TITLE:

\title{
Primitive chain network simulations for entangled DNA solutions.
}

\section{AUTHOR(S):}

Masubuchi, Yuichi; Furuichi, Kenji; Horio, Kazushi; Uneyama, Takashi; Watanabe, Hiroshi; lanniruberto, Giovanni; Greco, Francesco; Marrucci, Giuseppe

\section{CITATION:}

Masubuchi, Yuichi ...[et al]. Primitive chain network simulations for entangled DNA solutions.. The Journal of chemical physics 2009, 131(11): 114906.

\section{ISSUE DATE:}

2009-09-21

URL:

http://hdl.handle.net/2433/91447

\section{RIGHT:}

(C) 2009 American Institute of Physics. This article may be downloaded for personal use only. Any other use requires prior permission of the author and the American Institute of Physics. 


\title{
Primitive chain network simulations for entangled DNA solutions
}

\author{
Yuichi Masubuchi, ${ }^{1,2, a)}$ Kenji Furuichi, ${ }^{3}$ Kazushi Horio, ${ }^{1}$ Takashi Uneyama, ${ }^{1,2}$ \\ Hiroshi Watanabe, ${ }_{1}^{1}$ Giovanni lanniruberto, ${ }^{4}$ Francesco Greco, ${ }^{5}$ and Giuseppe Marrucci ${ }^{4}$ \\ ${ }_{1}^{1}$ Institute for Chemical Research, Kyoto University, Gokasyo, Uji, Kyoto 611-0011, Japan \\ ${ }^{2}$ JST-CREST, Kyoto 611-0011, Japan \\ ${ }^{3}$ TOYOBO Co., Ltd., Shiga 520-0292, Japan \\ ${ }^{4}$ Dipartimento di Ingegneria Chimica, Università degli Studi di Napoli “Federico II," Piazzale Tecchio, \\ 80-80125 Napoli, Italy \\ ${ }^{5}$ Istituto di Ricerche sulla Combustione, CNR, Piazzale Tecchio, 80-80125 Napoli, Italy
}

(Received 8 July 2009; accepted 21 August 2009; published online 21 September 2009)

\begin{abstract}
Molecular theories for polymer rheology are based on conformational dynamics of the polymeric chain. Hence, measurements directly related to molecular conformations appear more appealing than indirect ones obtained from rheology. In this study, primitive chain network simulations are compared to experimental data of entangled DNA solutions [Teixeira et al., Macromolecules 40, 2461 (2007)]. In addition to rheological comparisons of both linear and nonlinear viscoelasticities, a molecular extension measure obtained by Teixeira et al. through fluorescent microscopy is compared to simulations, in terms of both averages and distributions. The influence of flow on conformational distributions has never been simulated for the case of entangled polymers, and how DNA molecular individualism extends to the entangled regime is not known. The linear viscoelastic response and the viscosity growth curve in the nonlinear regime are found in good agreement with data for various DNA concentrations. Conversely, the molecular extension measure shows significant departures, even under equilibrium conditions. The reason for such discrepancies remains unknown. () 2009 American Institute of Physics. [doi:10.1063/1.3225994]
\end{abstract}

\section{INTRODUCTION}

Consistently with the stress-optical rule, ${ }^{1}$ molecular theories for polymer rheology are traditionally constructed to go hand in hand with the conformational dynamics of polymers. ${ }^{2}$ In principle, however, theories on polymer dynamics are more severely tested directly against optical measurements rather than through rheology. For example, entangled polymer motion validating the tube picture has been detected by neutron spin echo, ${ }^{3}$ neutron scattering, ${ }^{4}$ nuclear magnetic resonance, ${ }^{5}$ fluorescent microscopy, ${ }^{6}$ dielectric relaxation, ${ }^{7}$ molecular dynamics simulations, ${ }^{8}$ etc. Perkins et $a l .{ }^{6}$ performed direct observation of stained DNA molecules in concentrated unstained DNA solutions by fluorescent microscopy. The experiment, consisting in dragging one end of the DNA molecule, showed that the motion of the trailed DNA chain was confined by a tubelike region. Similar observations employing different methods have also been reported, ${ }^{3,5,8}$ confirming the existence of a dynamic constraint on a length scale fully consistent with the entanglement molecular weight estimated from rheology. As a further example, Bent et al. ${ }^{4}$ used neutron scattering to observe the molecular deformation of polystyrene melts flowing through a contraction. They obtained quantitative agreement with the predictions of a molecular theory for both scattering and rheology and with the same value of parameters for both, al-

\footnotetext{
${ }^{a)}$ Author to whom correspondence should be addressed. Electronic mail: mas@ @scl.kyoto-u.ac.jp. Tel.: +81-774-38-3136. FAX: +81-774-38-3139.
}

though rheology can be dealt with more swiftly with the simplified ROLIE-POLY model $^{9}$ rather than with the complete one.

In spite of the above successes, there still exist situations where molecular theories for rheology appear unable to explain other observables related to conformational dynamics. Watanabe et al. ${ }^{10}$ performed dielectric measurements of entangled polyisoprene under steady shear and found out that the end-to-end relaxation of polyisoprene chains is insensitive to flow, even for shear rates falling in the shear-thinning region. This phenomenon has been examined via sliplink simulations ${ }^{11}$ with negative results, and the reason for the discrepancy between model and experiment is still unknown.

Teixeira et al. ${ }^{12}$ carried out rheological measurements and molecular observations by fluorescent microscopy on several entangled DNA solutions differing in DNA concentration. They showed that rheology, including viscosity growth curves in the nonlinear range, is well described by the ROLIE-POLY equation. ${ }^{9}$ However, with the same model parameters, the molecular extension measure in steady shear flow was overestimated, and the response in the startup of shear flow was predicted to be essentially stepwise as compared to a much more gradual rise observed in the experiment. Teixeira et $a l .{ }^{12}$ also presented distributions of the DNA-molecule extension measure, but those distributions could not be compared with theory because typically theories only predict average values, sometimes even resorting to preaveraging approximations. ${ }^{13}$

In this study, primitive chain network simulations are compared to the DNA data set obtained by Teixeira et al. ${ }^{12}$ 
Our simulations are based on a model where each chain slides through several sliplinks, with each sliplink also connecting two chains in the simulation box, so that the overall system becomes an impermanent network in threedimensional (3D) space. ${ }^{14}$ The model incorporates the established dynamics of entangled polymers, i.e., reptation, ${ }^{2,15}$ contour length fluctuations, ${ }^{16}$ and thermal ${ }^{17}$ and convective ${ }^{18}$ constraint release, to reproduce linear ${ }^{19-22}$ and nonlinear ${ }^{20,23-25}$ rheologies. Force balance on entanglements is one aspect of the model that makes it different from other sliplink models, ${ }^{26-29}$ and its effect on rheology has also been examined. ${ }^{24,25}$ However, the model has not been frequently tested against other measures reflecting chain conformation, and, in some tests so far made on dielectric responses, ${ }^{11,30}$ the primitive chain network simulation failed to quantitatively reproduce the experimental results. It is thus meaningful to test the model against other conformational measures to look for possibly missing (or wrongly accounted for) relaxation mechanisms. Specifically, it appears interesting to investigate conformational distributions, somehow reflecting molecular individualism. ${ }^{31}$

\section{MODEL}

Since we employ the code used in Refs. 21 and 22, and no further implementation has been made in the present study, readers already familiar with the model may skip this section. In the primitive chain network simulations, polymer chains form a network in 3D space. The network consists of nodes, chain strands, and dangling ends, which, respectively, represent entanglements (sliplinks), polymer segments in between consecutive sliplinks, and chain ends. At a node, two polymer chains are connected by a sliplink (binary entanglement assumption).

We trace the dynamics of the system by the following set of state variables: position $\mathbf{R}$ of the network nodes or of chain ends, number $n$ of monomers in network strands or in chain ends, and number $Z$ of strands (chain ends included) composing a polymer chain. Thus the conformation of each chain in the system is described by the position of its chain ends, by the ordered set of bond vectors of its strands, and by the corresponding set of number of monomers.

Calculations are performed with nondimensional equations by choosing as the unit of length $a=b \sqrt{ } n_{0}$ (where $b$ is Kuhn length and $n_{0}$ is the mean equilibrium value of $n$ ), as the unit of energy $k T$, and as the unit of time $\tau=a^{2} \zeta / 6 k T$ (where $\zeta$ is the friction coefficient of the node). The variables mentioned hereafter are in nondimensional form. The monomer number $n$ is also normalized by taking the ratio to $n_{0}$.

The node position $\mathbf{R}$ changes in time due to a drag force from the medium, to the tension in the chain strands converging in the node, to an osmotic force induced by density fluctuations, and to thermal agitation. Change with time of the node position $\mathbf{R}$ is then described by a Langevin equation of motion. In nondimensional form, it is written as

$$
\dot{\mathbf{R}}-\hat{\mathbf{e}} \cdot \mathbf{R}=\frac{1}{2} \sum_{i}^{4} \frac{\mathbf{r}_{i}}{n_{i}}-\frac{1}{3} \nabla \mu+\mathbf{F} .
$$

Here $\hat{\mathbf{e}}$ is the velocity gradient tensor and $\mathbf{r}$ is the strand end-to-end vector. The chemical potential $\mu$ is derived from the repulsive interaction among the chains that maintains a uniform density with small fluctuations. The corresponding free energy $A$ is written as

$$
A=\left\{\begin{array}{cl}
\varepsilon\left(\frac{\phi(\mathbf{R})}{\langle\phi\rangle}-1\right)^{2} & \text { for } \phi(\mathbf{R})>\langle\phi\rangle \\
0 & \text { for } \phi(\mathbf{R}) \leq\langle\phi\rangle .
\end{array}\right.
$$

Here $\phi(\mathbf{R})$ is the local strand density (evaluated in sub-boxes of dimension $1^{3}$ in the simulation box), and $\langle\phi\rangle$ is the average value over the system. We choose $\langle\phi\rangle=10$. The numerical constant $\varepsilon$ is set at 0.5 . The third term in the right hand side of Eq. (1) is the thermal random force obeying $\langle\mathbf{F}\rangle=\mathbf{0}$ and $\left\langle\mathbf{F}(t) \mathbf{F}\left(t^{\prime}\right)\right\rangle=2 \delta\left(t-t^{\prime}\right)$. The equation of motion for the position of chain ends is similar to Eq. (1) with different numerical coefficients due to differences in friction and number of connected strands.

The number of monomers in each strand, $n$, also changes due to chain sliding through sliplinks induced by forces similar to those considered in node motion. The rate of change of $n$ with time is written as

$$
\frac{\dot{n}}{\varphi}=\left(\frac{r_{i}}{n_{i}}-\frac{r_{i-1}}{n_{i-1}}\right)-\frac{1}{3} \nabla \mu+f .
$$

Note that Eq. (3) gives the rate of change of $n$ in the strand $i$ due to monomer transport from the neighboring strand $i-1$, and $\varphi$ is the local linear density of monomers along the chain given by

$$
\varphi=\frac{1}{2}\left(\frac{n_{i}}{r_{i}}+\frac{n_{i-1}}{r_{i-1}}\right) .
$$

In both Eqs. (3) and (4) $r$ is the magnitude of $\mathbf{r}$. The third term in the right hand side of Eq. (3) is the random force obeying $\langle f\rangle=0$ and $\left\langle f(t) f\left(t^{\prime}\right)\right\rangle=(2 / 3) \delta\left(t-t^{\prime}\right)$.

The number $Z$ of strands per chain changes in time due to creation and destruction of sliplinks triggered by chain ends. If the number of monomers at a chain end is less than a certain minimum value, the neighboring sliplink is removed, corresponding to disentanglement of the chain end and of its partner (constraint release). On the contrary, if the number of monomers at a chain end exceeds a maximum value, a new sliplink is created on the end segment by randomly hooking a surrounding strand. The adopted monomer number window is $1 / 2<n<3 / 2$.

\section{SIMULATIONS}

Simulations were performed in a periodic boundary box of size of $16^{3}$. Since the average strand density $\langle\phi\rangle$ is 10 (per unit volume), more than 40000 strands (i.e., more than 1000 chains for $Z=40$ ) are contained in the box. After equilibrating the system for a sufficiently long time in the quiescent state, shear deformations were applied by using the LeesEdwards boundary condition as well as by switching on the 


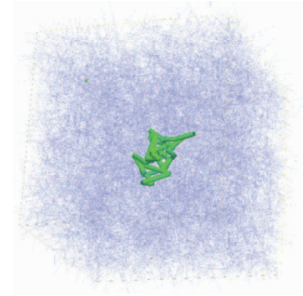

(a)

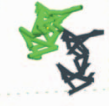

(b)

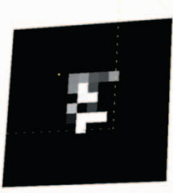

(c)
FIG. 1. Schematic representation of the procedure to obtain the $x$ measure. (a) A typical snapshot for the system with $Z_{0}=35$. A chain close to the center of the simulation box is highlighted, while thin lines show the segments of the other chains. (b) 2D projection of the highlighted 3D chain. (c) Intensity matrix obtained from the $2 \mathrm{D}$ projection.

velocity gradient tensor in Eq. (1). To obtain the linear viscoelastic response, we used oscillations of strain amplitude of 0.3 and calculated $G^{\prime}$ and $G^{\prime \prime}$ from the second cycle on. $^{21,22}$ The nondimensional stress tensor is calculated through the average $3\langle\mathbf{r r} / n\rangle$.

\section{A. The molecular extension measure}

The molecular extension measure $x$ was obtained by the following procedure, which mimics the DNA experiments performed under fluorescent microscopy as shown in Fig. 1. From the 3D chain conformation, the two-dimensional (2D) projection on the velocity-vorticity plane was obtained. Meshing that plane with a grid size of $1^{2}$, we count the number of nodes falling in each cell to obtain an intensity matrix. From the intensity matrix we calculate the maximum separation between the cells with a nonzero signal. Possible artifacts on our $x$ measure have been discussed previously, ${ }^{32,33}$ where it was shown that the choice of mesh size for the intensity matrix does not affect the relaxation behavior in linear and nonlinear viscoelastic regimes. Indeed, the relaxation of $x$ measure is essentially unchanged even if the $x$ measure is obtained as the maximum separation between the segments (not between the cells) in 3D space and in the $2 \mathrm{D}$ projection. It has been also reported that the reasonable number of chains for ensemble average is 1000 and thus the results shown later were obtained with this criterion.

\section{B. Parameters}

The parameters needed to map the calculated rheology onto the experimental data are a modulus $G_{0}$ and the time $\tau$. The modulus $G_{0}$ is related to $Z$ through the mean molecular weight $M_{0}$ of the network strand at equilibrium,

$$
G_{0}=\frac{\rho R T}{M_{0}}=\frac{\rho R T}{M} Z_{0} .
$$

Here $\rho$ is the polymer density, $M$ is the molecular weight of the polymer, and $Z_{0}=M / M_{0}$. According to Teixeira et al. ${ }^{12}$ $\rho=2.2 \mathrm{~kg} / \mathrm{m}^{3}$ for the highest DNA concentration $\left(\sim 31 \mathrm{C}^{*}\right)$ and $M=3.3 \times 10^{7}$ for $\lambda$-DNA. From these values, the best fit of the simulation results to the linear viscoelastic data determines $G_{0}, Z_{0}$, and $\tau$ for the $31 C^{*}$ solution (see Table I). To obtain the parameters for the other solutions, we employ the dilution relationship, ${ }^{17} G_{0}(\rho) \propto \rho^{d}$ and $Z_{0}(\rho) \propto \rho^{d-1}$, with a dilution exponent $d$ chosen at 2.2. In addition, assuming that the Rouse time of DNA is identical in all the solutions, we determine $\tau$ from the scaling $\tau(\rho) \propto \rho^{2-2 d}$. No additional parameter is introduced for the prediction of the viscosity growth curves, including those at high shear rates. All parameters relevant for rheology are summarized in Table I. Note that the value of $G_{0}$ obtained by the simulations is larger than the plateau modulus $G_{N}^{(0)}$ as is usually defined due to Brownian fluctuations of the nodes. ${ }^{19,21}$

Moving from rheology to the molecular extension measure $x$, also the unit of length $a$ is required. This is written as

$$
a=b \sqrt{n_{0}}=\sqrt{\frac{b L}{Z}},
$$

where $L$ is the contour length of the polymer, $L=22 \mu \mathrm{m}$ for $\lambda$-DNA. ${ }^{12}$ Values of $b$ of the order of $100 \mathrm{~nm}$ for DNA in solution have been reported in the literature. ${ }^{34,35}$ In the following we will use $b=130 \mathrm{~nm}$ (adopted by Teixeira et al. ${ }^{12}$ ) as well as other possible values, somehow treating $b$ as an adjustable parameter.

\section{RESULTS AND DISCUSSION}

Figure 2 compares linear viscoelastic data from Teixeira et $a l^{12}$ with simulation results obtained with the parameters reported in Table I. The primitive chain network model appears to capture the experimental response quantitatively. Particularly noteworthy is the fact that agreement at lower concentrations $\left(23 \mathrm{C}^{*}\right.$ and $\left.10 \mathrm{C}^{*}\right)$ is obtained by scaling the parameters from the values of the fit at $31 \mathrm{C}^{*}$ (see Sec. III B), rather than by direct data fitting at each concentration. Figure 2 , however, shows that the case of $16 \mathrm{C}^{*}$ is not reproduced by such scaling procedure, even when changing the dilution exponent $d$ in the range $2-2.5$. The reason for this peculiar discrepancy is unknown, and all $16 \mathrm{C}^{*}$ data are discarded hereafter.

TABLE I. Parameters used for simulations.

\begin{tabular}{lccccc}
\hline \hline Code & $\begin{array}{c}\rho \\
\left(\mathrm{kg} / \mathrm{m}^{3}\right)\end{array}$ & $Z_{0}$ & $\begin{array}{c}M_{0} \\
(\mathrm{~g} / \mathrm{mol})\end{array}$ & $\begin{array}{c}G_{0} \\
(\mathrm{~Pa})\end{array}$ & $\begin{array}{c}\tau \\
(\mathrm{s})\end{array}$ \\
\hline $10 \mathrm{C}^{*}$ & 0.65 & 10 & $3.4 \times 10^{6}$ & 5.6 & $1.5 \times 10^{-1}$ \\
$16 \mathrm{C}^{*}$ & 1.0 & 18 & $1.9 \times 10^{6}$ & 2.9 & $4.0 \times 10^{-2}$ \\
$23 \mathrm{C}^{*}$ & 1.5 & 26 & $1.3 \times 10^{6}$ & 1.3 & $1.6 \times 10^{-2}$ \\
$31 \mathrm{C}^{*}$ & 2.2 & 35 & $9.5 \times 10^{5}$ & 0.47 & $7.2 \times 10^{-3}$ \\
\hline \hline
\end{tabular}




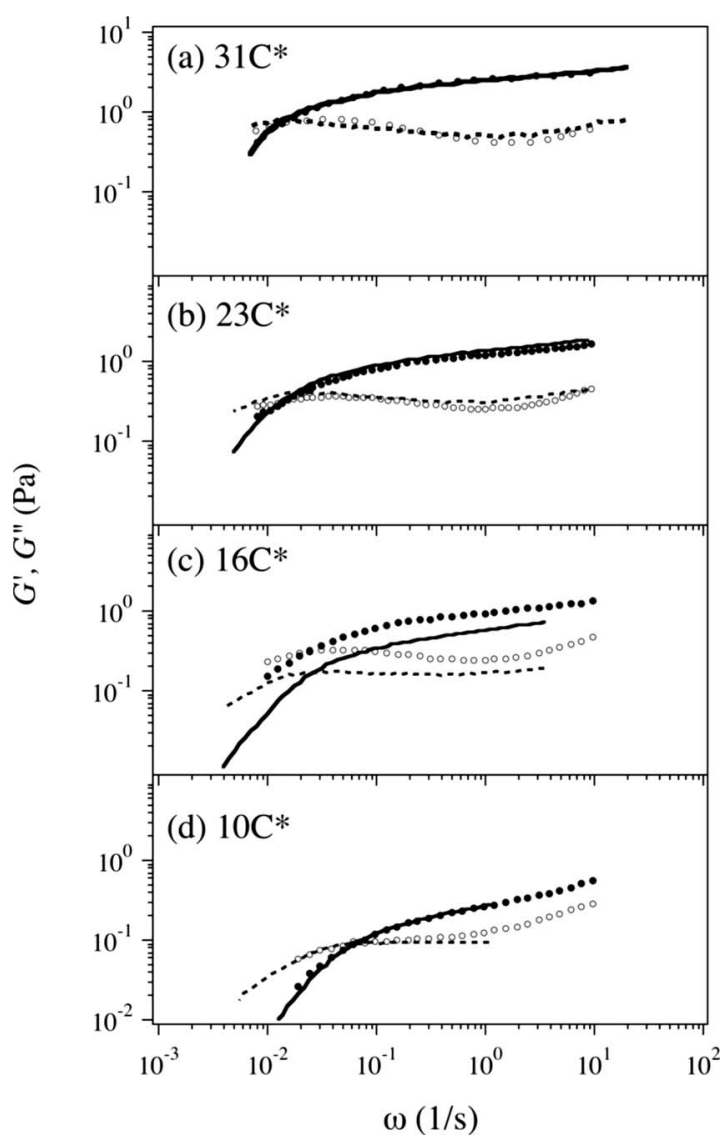

FIG. 2. Linear viscoelasticity of DNA solutions at four concentrations. Filled and unfilled circles are data from Ref. 12, while solid and broken curves are simulation results for $G^{\prime}$ and $G^{\prime \prime}$, respectively.

Figures 3 and 4 show the rheological response of the DNA solutions in shear flow. Figure 3 reports the viscosity growth curve in shear startup. (Results are reported only up to shear rates not exceeding $1 / \tau$, which is the inverse of our "unit" time, also marking the coarse-graining level of the primitive chain model.) In all cases reported in Fig. 3, the transient behavior is reasonably well captured, including the position of the maximum in the viscosity curve, whereas the steady-state values appear somewhat overestimated.

Figure 4 shows the steady shear stress of the $31 \mathrm{C}^{*}$ solution, in the form reported by Teixeira et al., ${ }^{12}$ which also includes the predictions of the ROLIE-POLY model ${ }^{9}$ (dotted line). Our simulations (full line), although overestimating the steady values, at least show a similar plateau as indicated by the data. A better way of accounting for convective constraint release ${ }^{18}$ in our simulations with respect to the simplified ROLIE-POLY equation might perhaps explain the difference. To be fair, it is noted that a better agreement between data and the ROLIE-POLY model might be obtained by artificially tuning the parameters of the ROLIEPOLY equation. In particular, the plateau indicated by the data could perhaps be predicted by the ROLIE-POLY equation by choosing small values of the Rouse time, which, however, would be inconsistent with the number of entanglements indicated by the linear viscoelastic response. ${ }^{12}$

Moving on to consider the molecular extension measure $x$, we first examined the equilibrium distribution of such

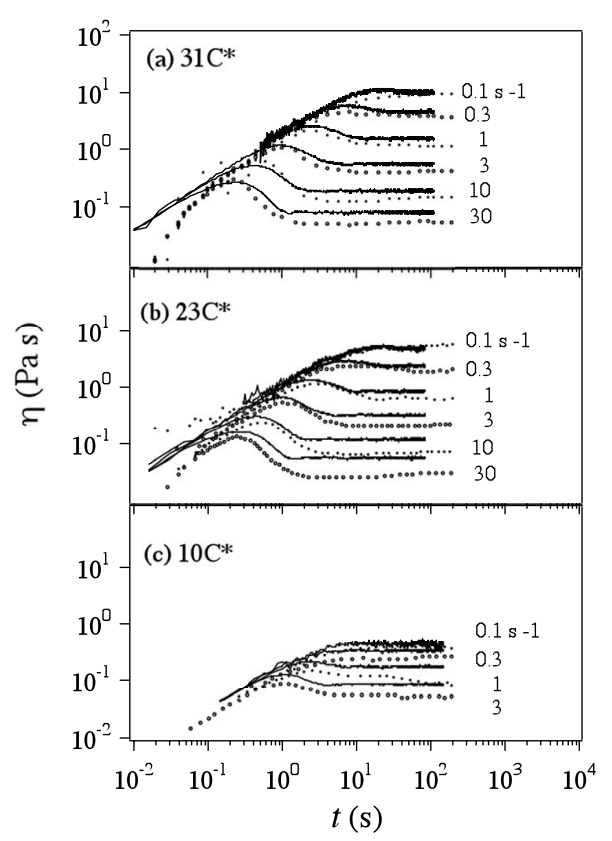

FIG. 3. Viscosity growth curves of DNA solutions at three concentrations. Symbols are data from Ref. 12 at the indicated shear rates, and curves are the corresponding simulation results.

quantity for the $31 \mathrm{C}^{*}$ solution. Figure 5 shows the prediction of the simulation (obtained with $b=130 \mathrm{~nm}$ ) and the experimental distribution, which we derived from Fig. 9(A) of Teixeira $e t a l .{ }^{12}$ where fluctuations of $x$ at equilibrium are reported for a time span of 250 s, i.e., for a period much longer than the slowest relaxation time. One should note that, although the equilibrium mean value of $x$ is well predicted by the simulation $(\langle x\rangle \approx 2 \mu \mathrm{m})$, the experimental distribution appears significantly more peaked than that resulting from the simulation. In order to check the quality of the primitive chain simulation results, we implemented a much simpler (unentangled) 3D random flight simulation with $Z$ $=35$ steps. As expected from theory (entanglements should not affect static properties), Fig. 5 shows that the two simulations are essentially equivalent in predicting the equilibrium $x$ distribution. It should also be mentioned that the observed discrepancy in Fig. 5 between data and simulation cannot be resolved by changing the value of the $b$ parameter.

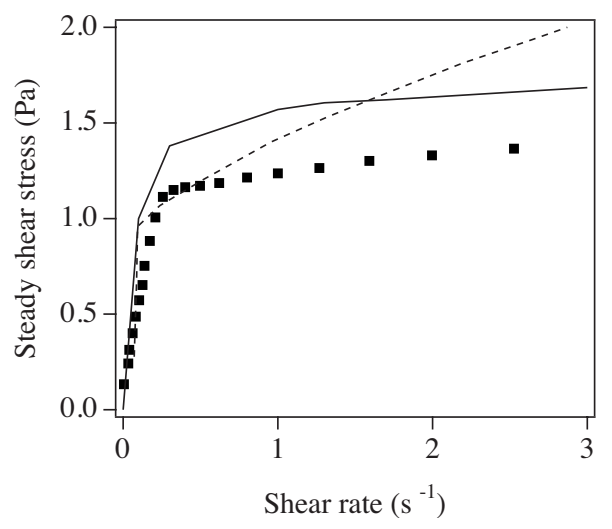

FIG. 4. Steady shear stress as a function of shear rate for the $31 \mathrm{C}^{*}$ solution. Symbols are data from Ref. 12. Solid and broken curves are predictions of our model and of the ROLIE-POLY model, respectively. 


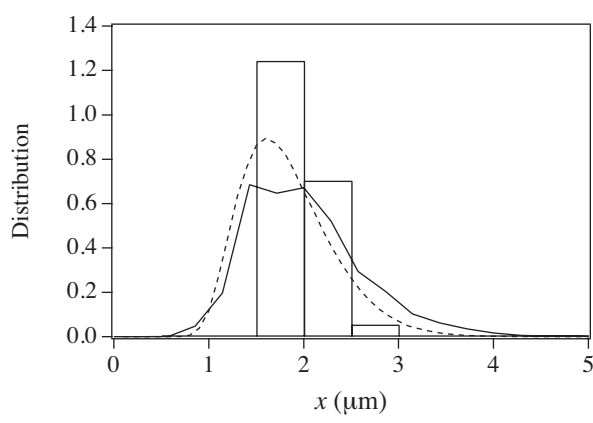

FIG. 5. Equilibrium distribution of the molecular extension $x$ for the $31 \mathrm{C}^{*}$ DNA solution. Histogram is obtained from the $x$ fluctuations appearing in Fig. 9A of Ref. 12. Solid and broken curves are from primitive chain, and unentangled Gaussian chain simulations, respectively.

Indeed, by decreasing $b$, the predicted distribution curve would become more peaked but it would also shift to the left in Fig. 5, the mean value of $x$ (roughly the peak of the curve) departing from that of the data.

Figures 6 and 7 show $x$ results in steady shear for the same $\left(31 C^{*}\right)$ solution. Figure 6 reports average values as a function of shear rate. The data are from Teixeira et al. ${ }^{12}$ while the curves are simulation results for different values of $b$, ranging from 130 down to $30 \mathrm{~nm}$. In the simulations, the average was taken from more than 1000 chains. Figure 7 shows distributions, obtained by using 10000 chains, at shear rates of $0.3 \mathrm{~s}^{-1}$ [Fig. 7(a)] and $2.7 \mathrm{~s}^{-1}$ [Fig. 7(b)]. By ignoring that the equilibrium results confirmed a $b$ value of $130 \mathrm{~nm}$, the best fit with data of both averages and distributions in Figs. 6 and 7 is obtained by choosing $b=65 \mathrm{~nm}$.

Figure 8 extends the comparison between measured and calculated $x$ distributions in flow to the other DNA concentrations. In Fig. 8(a), the adopted $b$ value is $65 \mathrm{~nm}$. Agreement is fair for the case of $23 C^{*}$ (just as for $31 C^{*}$ ), whereas a significant discrepancy is observed at the lowest concentration $\left(10 C^{*}\right)$. In Fig. 8(b), the adopted $b$ value is $130 \mathrm{~nm}$. Contrary to Fig. 8(a), agreement is now better for the low concentration $\left(10 \mathrm{C}^{*}\right)$ and worse for the higher ones. It is also noteworthy that the experimentally obtained distribution is rather insensitive to the concentration (at any assigned shear rate) as if relaxation times were not affected, although linear rheology shows the opposite (see Fig. 2). On the contrary,

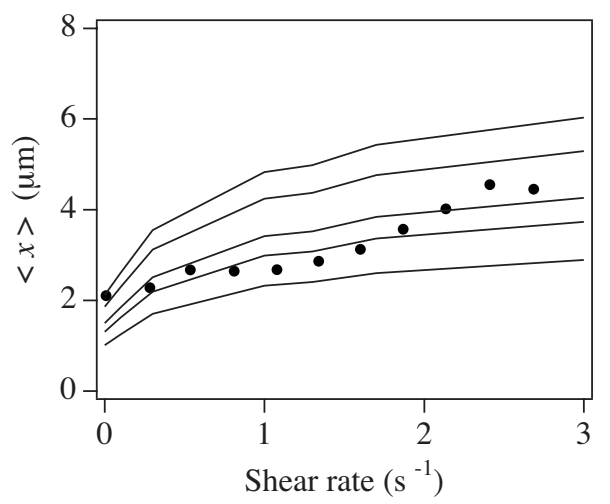

FIG. 6. Average value of $x$ at steady state as a function of shear rate for the $31 C^{*}$ solution. Symbols are data from Fig. 15B of Ref. 12. Curves are simulation results for various Kuhn step lengths: $b=130,100,65,50$, and 30 $\mathrm{nm}$, from top to bottom.
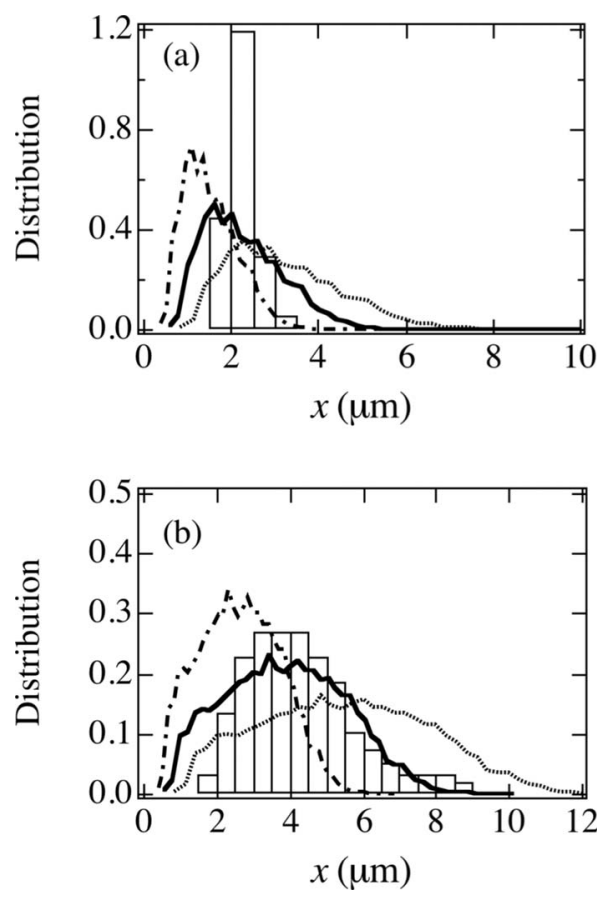

FIG. 7. Distribution of $x$ for the $31 \mathrm{C}^{*}$ solution in steady shear at (a) $0.3 \mathrm{~s}^{-1}$ and (b) $2.7 \mathrm{~s}^{-1}$. Histograms are data from Ref. 12. Dashed, solid, and dotted curves are simulation results for $b=30,65$, and $130 \mathrm{~nm}$, respectively.

simulations remain sensitive to change in relaxation time with concentration, which is particularly apparent in the shift to the left of the simulation curves at $10 \mathrm{C}^{*}$ in Fig. 8(a), and in the shift to the right of the curves for $23 \mathrm{C}^{*}$ and $31 \mathrm{C}^{*}$ in

(a)

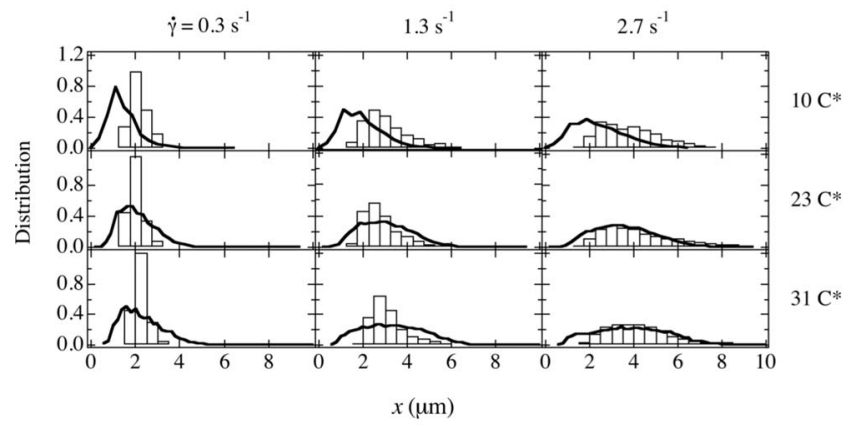

(b)

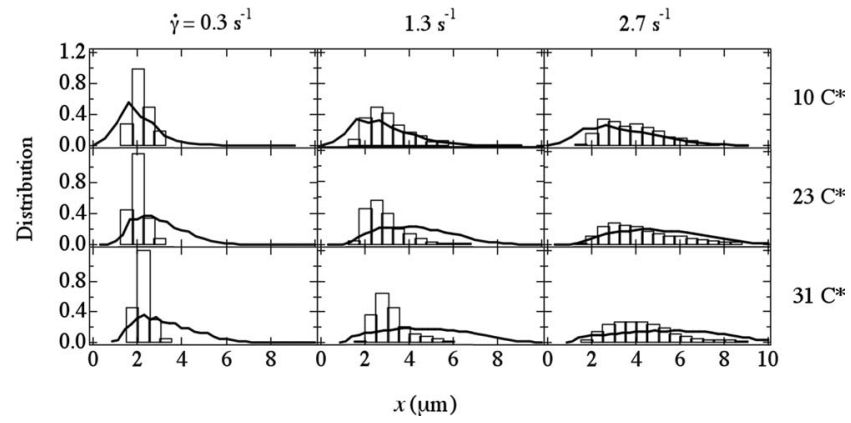

FIG. 8. Distribution of $x$ for several concentrations (one per row) at various shear rates (one per column). Histograms are from Ref. 12. Curves are simulation results over samples of 10000 chains. In (a) $b=65 \mathrm{~nm}$ was used; in (b) Kuhn length is $130 \mathrm{~nm}$. 
Fig. 8(b). Similar results on the conformational distribution have been reported for another multichain sliplink model recently developed by Dambal et al. ${ }^{36}$

Figure 9 reports the transient behavior of $x$ following the startup of a shear flow for the $31 \mathrm{C}^{*}$ solution and compares it with the corresponding behavior of the transient viscosity. The $x$ simulation results are obtained with $b=130 \mathrm{~nm}$. Figure 9 (a) shows that the viscosity curve predicted by the simulation is similar to data both at low and high shear rates [see also Fig. 3(a)]. Conversely, Fig. 9(b) reveals that the experimental $x$ rise with time (at a shear rate of $1 \mathrm{~s}^{-1}$ ) is much slower than predicted by simulation. Indeed, while simulations predict a similar kinetics for both rheology and conformation, data do not confirm such behavior, as is immediately apparent by comparing data in Figs. 9(a) and 9(b).

The numerous discrepancies observed between model and experiments concerning the $x$ measure seem hard to interpret, especially in an all-comprehensive way. Starting from the equilibrium data in Fig. 5, it should preliminarily be mentioned that our procedure for extracting the data from Fig. 9A in Ref. 12 might have introduced some errors. If taken for granted, however, those data indicate that the equilibrium DNA molecule is not Gaussian, possibly due to the influence of electrical charges not fully screened or because of other (unknown) interactions. The behavior of $x$ in steady shear (Fig. 6), showing a modest increase with increasing shear rate, could be due either to the effect of these restraining forces (electric or otherwise) or, as suggested by Teixeira et al. ${ }^{12}$ to a mysterious effect which makes the $x$ kinetics to correlate with the Rouse time (equal to approximately $2 \mathrm{~s}$, independently of concentration) rather than with the time of disengagement from the entangled network (equal to approximately $90 \mathrm{~s}$ for the $31 \mathrm{C}^{*}$ solution). Possible (mysterious) dependence of $x$ behavior on Rouse time would also agree with the fact that distributions appear insensitive to concentration (Fig. 8). However, the transient response during startup of shear flow (Fig. 9) indicates a time to reach a (weakly deformed) steady state much longer than Rouse time. Apart from the contradictions of the different behaviors here summarized, none of the above hypotheses would explain why the $x$ measure should be profoundly affected by some unknown extra "force" while the rheology remains unaffected. Hence the only possible conclusion as of today seems that the physical origin of the observed behavior is not understood.

In relation to our previous work, ${ }^{10,11,30}$ however, further comments on the observed time of the transient response seem in order. In Fig. 10 we show the transient response of the $x$ measure for various shear rates as obtained from simulations with $b=130 \mathrm{~nm}$, to be compared with the single available data set previously reported in Fig. 9(b). In Fig. 10(a) we show absolute values, whereas in Fig. 10(b) we normalize $x$ to the difference between steady-state and equilibrium values. The experimental data (at $1.0 \mathrm{~s}^{-1}$ ) indicate a characteristic time of approximately $90 \mathrm{~s}$ [close to the longest relaxation time of linear viscoelasticity, see Figs. 2(a) and 3(a)]. On the other hand, the simulations predict a faster relaxation at the identical shear rate as a result of acceleration due to shear, as shown in Fig. 10(b). Figure 10(b) also

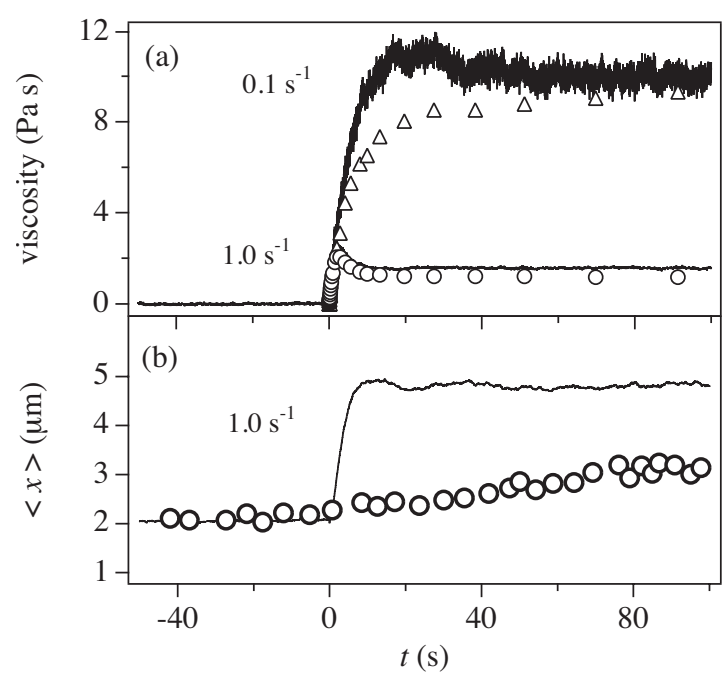

FIG. 9. Transient response of (a) viscosity and (b) average molecular extension $\langle x\rangle$ in startup of shear flow for the $31 \mathrm{C}^{*}$ solution at the indicated shear rates. Symbols are data from Ref. 12 at $1.0 \mathrm{~s}^{-1}$ (circle) and $0.1 \mathrm{~s}^{-1}$ (triangle). Curves are simulation results with $b=130 \mathrm{~nm}$.

shows that the normalized transient response of the experiment is similar to that predicted by simulations at the shear rate of $0.03 \mathrm{~s}^{-1}$, which is close to the linear regime. (In our simulation box, fluctuations that are already significant at $0.03 \mathrm{~s}^{-1}$ completely mask the transient startup in the linear regime.) Although absolute values do not match [see Fig. 10(a)], it so appears that the characteristic time of the $x$ re-
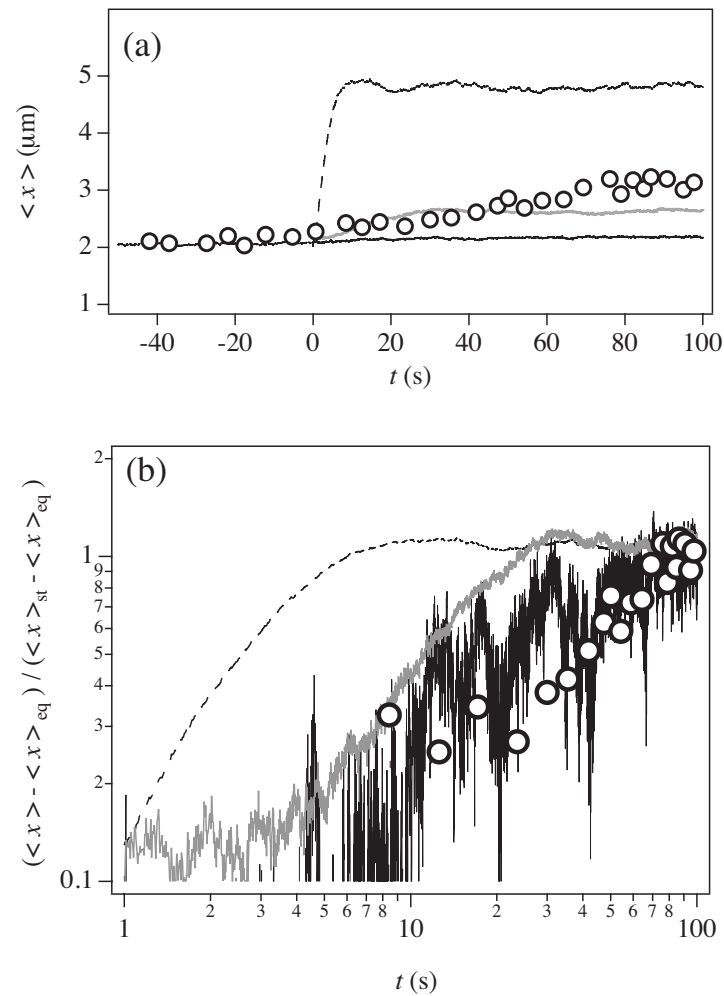

FIG. 10. Transient response of the average molecular extension $\langle x\rangle$ in startup of shear flows for the $31 \mathrm{C}^{*}$ solution. (a) Curves are simulation results for $1.0 \mathrm{~s}^{-1}$ (dotted curve), $0.1 \mathrm{~s}^{-1}$ (hatched curve), and $0.03 \mathrm{~s}^{-1}$ (solid curve); symbols are data at $1.0 \mathrm{~s}^{-1}$ from Ref. 12. (b) Same as in (a), normalized to the difference between steady and equilibrium values (denoted by $\langle x\rangle_{\mathrm{st}}$ and $\left.\langle x\rangle_{\mathrm{eq}}\right)$. 
sponse in the nonlinear regime remains roughly equal to the value of the linear regime. Thus it is reasonably supposed that the $x$ response in the experiment is not accelerated by shear. As mentioned earlier, a similar insensitivity to shear flow has been reported for another conformational measure, namely, the end-to-end distance as observed in dielectric measurements. ${ }^{10}$ For both cases, simulations predict a decrease in the conformational characteristic time with increasing shear rate, which is denied by data. In the nonlinear range the acceleration of relaxation is mainly induced by convective constraint release, and such acceleration is indeed proven by rheology as revealed, for example, by the decrease in the time needed to reach steady state in Figs. 3 and 9(a). Reduction in the effective relaxation time in steady shear flow is also proven by oscillatory data in parallel superposition (see, e.g., Ref. 37 and the literature cited therein). On the contrary, Figs. 9(b) and 10 seem to indicate that no such acceleration applies to conformational properties. Hence we plan to investigate possible changes in the simulation model, for example, in the rules by which chain end dynamics renew the network topology. The aim would be to somehow uncouple local rearrangements that are relevant to rheology from global changes that determine the overall chain conformation. Further experiments on conformational properties at several shear rates would also be welcome.

\section{CONCLUSIONS}

Primitive chain network simulations were performed for entangled DNA solutions of different concentrations and the results compared with rheological and molecular extension data recently reported by Teixeira et al. ${ }^{12}$ The linear viscoelasticity and the viscosity growth during startup of shear flows at several shear rates were quantitatively reproduced. The parameters needed for such a comparison, namely, a modulus and a time, were determined for the most concentrated solution $\left(31 \mathrm{C}^{*}\right)$, and then scaled with concentration by using well established dilution power laws. (It should be mentioned, however, that for the DNA solution with concentration $16 \mathrm{C}^{*}$ quantitative agreement was not achieved for unknown reasons.)

Concerning the molecular extension measure $x$, on the contrary, the overall comparison between data and simulations proved unsatisfactory, although agreement could be found in some cases, often by suitably adjusting the single parameter that needs to be assigned for conformational comparisons, i.e., the Kuhn segment length $b$. Indeed, the equilibrium $x$ measure could be fitted by using the independently determined $b$ value of $130 \mathrm{~nm}$, while $x$ values in fast steady shear flows appeared to require $b=65 \mathrm{~nm}$.

As regards $x$ distributions, the equilibrium and slow flow experimental distributions appear more peaked than those obtained from simulations. It should be mentioned, however, that the equilibrium distribution obtained from the simulations agrees with the classical notion that entangled chains are Gaussian, a fact that the equilibrium data on these DNA solutions (although meager) do not seem to confirm. Good agreement is found in fast flows, although with $b=65 \mathrm{~nm}$ for the $23 \mathrm{C}^{*}$ and $31 \mathrm{C}^{*}$ solutions and with $b=130 \mathrm{~nm}$ for the $10 C^{*}$ solution.

The transient behavior of the molecular extension in startup of shear flows revealed yet another significant discrepancy, insofar as the simulation predicts an essentially stepwise upward response while data show a very gradual growth toward the steady value. So far, we were unable to find any consistent interpretation for these discrepancies.

\section{ACKNOWLEDGMENTS}

This work was partly supported by CREST of the Japan Science and Technology Agency and by the Ministry of Education, Science, Sports and Culture of Japan through the Grant-in-Aid for Scientific Research (B), Grant No. 20340111, 2008.

${ }^{1}$ H. Janeschitz-Kriegl, Polymer Melt Rheology and Flow Birefringence (Springer, Berlin, 1983).

${ }^{2}$ M. Doi and S. F. Edwards, The Theory of Polymer Dynamics (Clarendon, Oxford/Oxford University Press, New York, 1986).

${ }^{3}$ D. Richter, B. Ewen, B. Farago, and T. Wagner, Phys. Rev. Lett. 62, 2140 (1989).

${ }^{4}$ J. Bent, L. R. Hutchings, R. W. Richards, T. Gough, R. Spares, P. D. Coates, I. Grillo, O. G. Harlen, D. J. Read, R. S. Graham, A. E. Likhtman, D. J. Groves, T. M. Nicholson, and T. C. B. McLeish, Science 301, 1691 (2003).

${ }^{5}$ M. E. Komlosh and P. T. Callaghan, J. Chem. Phys. 109, 10053 (1998).

${ }^{6}$ T. T. Perkins, D. E. Smith, and S. Chu, Science 264, 819 (1994).

${ }^{7}$ H. Watanabe, Prog. Polym. Sci. 24, 1253 (1999).

${ }^{8}$ K. Kremer and G. S. Grest, J. Chem. Phys. 92, 5057 (1990).

${ }^{9}$ A. E. Likhtman and R. S. Graham, J. Non-Newtonian Fluid Mech. 114, 1 (2003).

${ }^{10} \mathrm{H}$. Watanabe, S. Ishida, and Y. Matsumiya, Macromolecules 35, 8802 (2002).

${ }^{11}$ Y. Masubuchi, H. Watanabe, G. Ianniruberto, F. Greco and G. Marrucci, Nihon Reoroji Gakkaishi 32, 197 (2004).

${ }^{12}$ R. E. Teixeira, A. K. Dambal, D. H. Richter, E. S. G. Shaqfeh, and S. Chu, Macromolecules 40, 2461 (2007).

${ }^{13}$ T. C. B. McLeish, Adv. Phys. 51, 1379 (2002).

${ }^{14}$ Y. Masubuchi, J. I. Takimoto, K. Koyama, G. Ianniruberto, G. Marrucci, and F. Greco, J. Chem. Phys. 115, 4387 (2001).

${ }^{15}$ P. G. de Gennes, J. Chem. Phys. 55, 572 (1971)

${ }^{16}$ M. Doi, J. Polym. Sci., Part C: Polym. Lett. 19, 265 (1981).

${ }^{17}$ W. W. Graessley, Adv. Polym. Sci. 47, 67 (1982).

${ }^{18}$ G. Marrucci, J. Non-Newtonian Fluid Mech. 62, 279 (1996).

${ }^{19}$ Y. Masubuchi, G. Ianniruberto, F. Greco, and G. Marrucci, J. Chem. Phys. 119, 6925 (2003).

${ }^{20}$ Y. Masubuchi, G. Ianniruberto, F. Greco, and G. Marrucci, Modell. Simul. Mater. Sci. Eng. 12, S91 (2004).

${ }^{21}$ Y. Masubuchi, G. Lanniruberto, F. Greco, and G. Marrucci, J. NonNewtonian Fluid Mech. 149, 87 (2008).

${ }^{22}$ Y. Masubuchi, H. Watanabe, G. Ianniruberto, F. Greco, and G. Marrucci, Macromolecules 41, 8275 (2008).

${ }^{23}$ T. Yaoita, T. Isaki, Y. Masubuchi, H. Watanabe, G. Ianniruberto, F. Greco, and G. Marrucci, J. Chem. Phys. 128, 154901 (2008).

${ }^{24}$ K. Furuichi, C. Nonomura, Y. Masubuchi, G. Ianniruberto, F. Greco, and G. Marrucci, Nihon Reoroji Gakkaishi 35, 73 (2007).

${ }^{25}$ K. Furuichi, C. Nonomura, Y. Masubuchi, H. Watanabe, G. Ianniruberto, F. Greco, and G. Marrucci, Rheol. Acta 47, 591 (2008).

${ }^{26}$ C. C. Hua and J. D. Schieber, J. Chem. Phys. 109, 10018 (1998).

${ }^{27}$ M. Doi and J.-I. Takimoto, Philos. Trans. R. Soc. London, Ser. A 361, 641 (2003).

${ }^{28}$ J. D. Schieber, J. Neergaard, and S. Gupta, J. Rheol. 47, 213 (2003).

${ }^{29}$ A. E. Likhtman, Macromolecules 38, 6128 (2005).

${ }^{30}$ T. Uneyama, Y. Masubuchi, K. Horio, Y. Matsumiya, H. Watanabe, J. A. Pathak, and C. M. Roland, J. Polym. Sci., Part B: Polym. Phys. 47, 1039 (2009).

${ }^{31}$ P. G. de Gennes, Science 276, 1999 (1997). 
${ }^{32}$ Y. Masubuchi, H. Watanabe, G. Ianniruberto, F. Greco and G. Marrucci, Nihon Reoroji Gakkaishi 36, 181 (2008).

${ }^{33}$ Y. Masubuchi, T. Uneyama, H. Watanabe, G. Ianniruberto, F. Greco, and G. Marrucci, Nihon Reoroji Gakkaishi 37, 65 (2009).

${ }^{34}$ J. B. Hays, M. E. Magar, and B. H. Zimm, Biopolymers 8, 531 (1969).
${ }^{35}$ D. Porschke, Biophys. Chem. 40, 169 (1991).

${ }^{36}$ A. Dambal, A. Kushwaha and E. S. G. Shaqfeh, Macromolecules, DOI:10.1021/ma802555r (2009).

${ }^{37}$ E. Somma, O. Valentino, G. Titomanlio, and G. Ianniruberto, J. Rheol. 51, 987 (2007). 